\title{
Chiral magnetic interactions in graphene nanoribbons on topological insulator substrates
}

\author{
Wei Zhang, ${ }^{1}$ Farideh Hajiheidari, ${ }^{2}$ and Riccardo Mazzarello ${ }^{2,3, *}$ \\ ${ }^{1}$ Center for Advancing Materials Performance from the Nanoscale, State Key Laboratory for Mechanical Behavior of Materials, \\ $X i$ 'an Jiaotong University, Xi'an 710049, PR China \\ ${ }^{2}$ Institute for Theoretical Solid State Physics, RWTH Aachen University, D-52074 Aachen, Germany \\ ${ }^{3}$ JARA-FIT and JARA-HPC, RWTH Aachen University, D-52074 Aachen, Germany
}

(Received 26 April 2017; published 15 December 2017)

\begin{abstract}
It is known that the interplay between strong spin-orbit coupling and broken inversion symmetry can lead to chiral configurations in low-dimensional magnetic systems deposited on heavy-element surfaces. This phenomenon is due to the Dzyaloshinskii-Moriya interaction, which favours noncollinear magnetic structures. Here we show by $a b$ initio simulations that this interaction leads to a twisting of the two antiferromagnetically coupled edge-state spins of a zigzag graphene nanoribbon deposited on the topological insulator $\mathrm{Sb}_{2} \mathrm{Te}_{3}$. To our knowledge, this is the first report of a chiral magnetic structure formed by a pair of one-dimensional states. This effect results in a finite net magnetization in the nanoribbons, which could lead to applications such as spin filters in graphene-based spintronics devices.
\end{abstract}

DOI: 10.1103/PhysRevB.96.245413

\section{INTRODUCTION}

The surface states of three-dimensional topological insulators (TIs) exhibit several remarkable properties, which stem from time-reversal symmetry and strong spin-orbit coupling (SOC) [1-5]. These properties include spin-momentum locking and robustness against time-reversal-invariant perturbations, such as nonmagnetic impurities: as a result, the surface states are immune to disorder-induced localization. Furthermore, the surface states strongly affect the exchange coupling between magnetic impurities deposited on the surface $[6,7]$. More specifically, they are predicted to induce a strong anisotropic Dzyaloshinskii-Moriya interaction (DMI) [8-13] between the spins of pairs of magnetic adatoms, which favours noncollinear magnetic structures [7]. In these systems, the magnitude of the DMI is comparable to that of the conventional Ruderman-Kittel-Kasuya-Yoshida (RKKY) indirect exchange interaction [7]. On the other hand, magnetic impurities and, more generally, perturbations destroying time-reversal symmetry affect the surface state properties dramatically. In particular, they can open an energy gap and induce an anomalous quantum Hall effect [4,5,14-17]. The proximity of a TI surface to a magnetic system containing a domain wall can also lead to the formation of one-dimensional chiral states $[4,5,17]$.

Here it is shown by $a b$ initio simulations based on density functional theory (DFT) that the DMI leads to a twisting of the two antiferromagnetically coupled edge-state spins of a zigzag-terminated graphene nanoribbon (GNR) [18-24] deposited on the (111) surface of the TI $\mathrm{Sb}_{2} \mathrm{Te}_{3}$ [25], and this novel phenomenon stems from the proximity to a substrate with strong SOC. Zigzag GNRs were predicted to display magnetic electronic states localized at the edge several years before graphene was produced experimentally [18]. Computational [26] and experimental [27,28] (albeit indirect) evidence for the presence of edge magnetism in GNRs deposited on gold has been recently provided. Experimental

\footnotetext{
*mazzarello@physik.rwth-aachen.de
}

progress has been hindered by difficulties in controlling the edge geometry and chemistry, and by the fact that direct measurements of the tiny magnetic moments are not possible. In this paper, we focus on unpassivated edges, which bind strongly to the substrate. In contrast, monohydrogenated GNRs have been shown to interact with $\mathrm{Sb}_{2} \mathrm{Te}_{3}$ weakly [29], similarly to the behavior of perfect graphene [30]. In spite of the weak interaction, the proximity effects of substrates with large SOC have been shown to alter the properties of pure graphene significantly, which is of interest for spintronics applications [30-34].

\section{METHODS}

The structural optimizations and the calculations of the electronic and magnetic properties are carried out using the plane-wave package Quantum-Espresso [35]. We employ generalized gradient approximation functionals [36] with the semi-empirical van der Waals corrections by Grimme [37] to perform structural relaxation, without including SOC. During the optimization, the two topmost layers of the substrate are allowed to relax. The SOC simulations and the calculations of the energy differences between magnetic structures are carried out using local-density-approximation [38] functionals. A similar approach exploiting the strength of different exchange functionals was used in Ref. [39]. We employ (scalar relativistic and fully relativistic) norm-conserving pseudopotentials. Wave functions are expanded in plane waves with an energy cutoff of 60 Ry.

We focus on the (111) surface of $\mathrm{Sb}_{2} \mathrm{Te}_{3}$, and consider slabs of $\mathrm{Sb}_{2} \mathrm{Te}_{3}$ containing 15 atomic layers (three quintuple layers). The GNR has a width of six graphene units and is deposited on the "top" surface of the slab, whereas the "bottom" surface is clean. Since the lattice constant of the $\mathrm{Sb}_{2} \mathrm{Te}_{3}(111)$ surface is exactly three times as large as that of graphene (and, thus, of a zigzag GNR unit) [29,30], $4.26 \AA$ versus $1.42 \AA$, we employ an orthorhombic supercell that contains three GNR units along the direction of the GNR [assumed to be parallel to the $y$ axis; see Fig. 1(b), where the blue rectangle indicates the supercell employed in the simulations]. The orthorhombic 


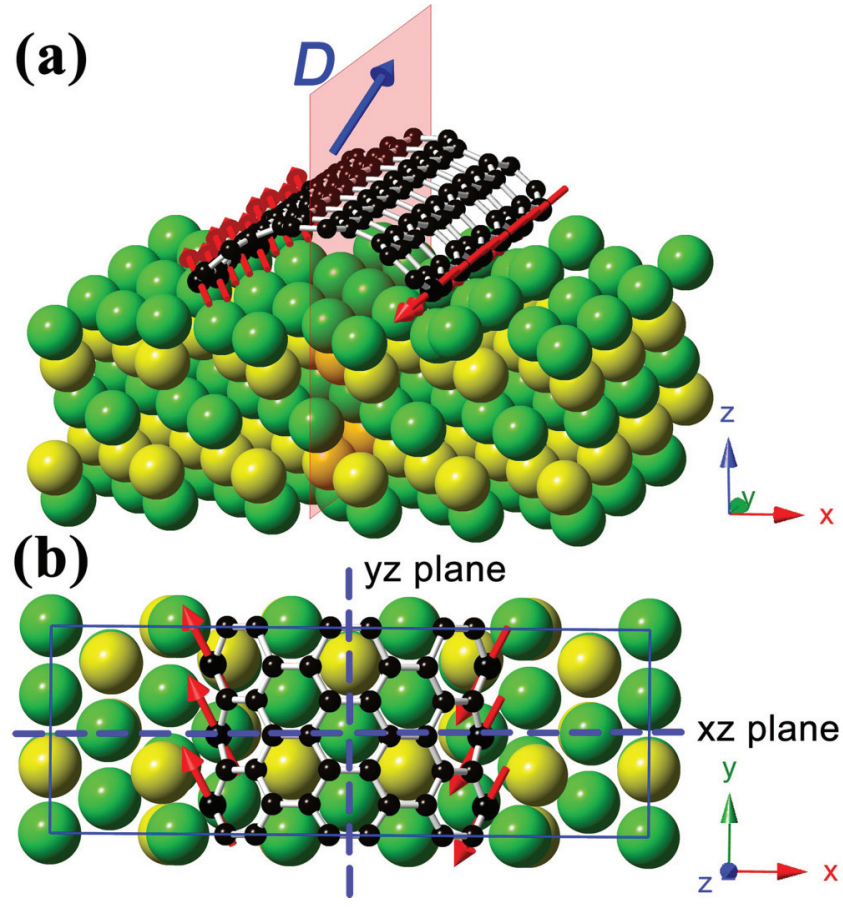

FIG. 1. Atomistic models. (a) Side and (b) top view of the GNR on $\mathrm{Sb}_{2} \mathrm{Te}_{3}(111)$ after relaxation. A thick slab of $\mathrm{Sb}_{2} \mathrm{Te}_{3}$ is considered in the DFT simulations, but only five atomic layers are shown here for the sake of clarity. The red arrows render the spin structure formed by the edge states in the lowest-energy configuration obtained. The directions of the edge magnetic moments deviate slightly from the $y$ axis due to the DMI. The blue dashed lines in (b) depict the $y z$ and $x z$ planes: the first one is an exact mirror plane, whereas the mirror symmetry with respect to the second one is broken by the atomic layers beneath the topmost Te layer of the substrate. The blue rectangle in (b) indicates the actual supercell employed in the simulations. In this and the following figure, $\mathrm{Sb}, \mathrm{Te}$, and $\mathrm{C}$ atoms are rendered with yellow, green, and black spheres, respectively.

supercell has lattice parameters $a=21.3 \AA, b=7.38 \AA$, $c=46 \AA$. The $c$ parameter corresponds to a vacuum layer of thickness $17 \AA$, which decouples the periodic images of the slab along the $z$ axis perpendicular to the surfaces. In total, the model contains 186 atoms. A $1 \times 8 \times 1$ Monkhorst-Pack mesh [40] is employed to perform the integration over the Brillouin zone. For the projected density of states, we use a Gaussian broadening of $0.014 \mathrm{eV}$.

\section{RESULTS}

Several adsorption configurations for the GNR are investigated, and the energetically most stable model is shown in Fig. 1. Upon geometry relaxation, the GNR bends considerably and forms chemical bonds at the edges to saturate the danglingbond orbitals of the edge $\mathrm{C}$ atoms. The distance between the edge $\mathrm{C}$ atom and $\mathrm{Sb}_{2} \mathrm{Te}_{3}(111)$ is $1.95 \AA$, while the distance between the center of the GNR and the surface is $4.72 \AA$ (further information is provided in the Supplemental Material [41]). Similar geometries are observed for $\mathrm{H}$-free nanoribbons on metal substrates $[26,42,43]$.
Remarkably, the magnetic properties of the GNR are mostly preserved, in spite of the strong chemical interaction at the edges. Preliminary spin-polarized calculations without SOC reveal that the GNR exhibits edge magnetism with antiferromagnetic (AFM) coupling between the two edges: the energy difference between the AFM and ferromagnetic (FM) (respectively, nonmagnetic) configuration is about $2.6 \mathrm{meV}$ (respectively, $7.8 \mathrm{meV}$ ). The magnetization per edge $\mathrm{C}$ atom is $0.33 \mu_{B}$, to be compared to the value of $0.34 \mu_{B}$ obtained for a freestanding GNR using an equivalent k-point mesh. The presence of spin-polarized edge states is in striking contrast to the case of unpassivated GNRs on metal substrates, wherein the edge magnetism is fully suppressed [26,42,43]. Inspection of the projected density of states (PDOS) and the plots of the charge density of relevant states (see Fig. S2) show that the chemical interaction between the GNR and $\mathrm{Sb}_{2} \mathrm{Te}_{3}$ mainly stems from the bonding between the $\sigma$-like orbitals of the edge $\mathrm{C}$ atoms and the $p$ states of the neighboring Te atoms. On the other hand, the $\pi$ orbitals of the edge $\mathrm{C}$ atoms forming the edge states hardly contribute to the bonding.

Next, we include SOC. The bonding mechanisms at the edge are not affected qualitatively. The corresponding nonspin-polarized PDOS for the $|j=3 / 2, l=1\rangle+\mid j=1 / 2$, $l=1\rangle$ spin-angle functions of the edge $\mathrm{C}$ atoms display a large peak at the Fermi energy $\mathrm{E}_{F}$ [see Figure 2(a)]. The edge states contribute significantly to the peak. Similarly to the case of freestanding GNRs, a Stoner instability occurs, which leads to edge magnetism. The resulting splitting of the spin-polarized PDOS peaks is about $0.16 \mathrm{eV}$, as shown in Fig. 2(b). Inclusion of SOC has additional remarkable effects on the magnetic structure, which originate from the interplay between magnetic anisotropies and chiral DMI. We perform three independent self-consistent calculations with initial direction of the AFM spin configuration along $x, y$, and $z$, respectively. Irrespective of the initial configuration, a small but non-negligible twisting of the spins of the two edge states occurs. As a result, the system acquires a finite net magnetization of the order of $0.2-0.3 \mu_{B}$, whose direction differs from the direction of the two individual magnetic moments. In Figs. 2(b)-2(d), we show the magnetic properties of the lowest energy configuration obtained [schematically depicted in Fig. 1(a)], in which the two moments have opposite $y$ and $z$ components (the latter being much smaller than the former), but also have a parallel component along $x$, resulting in an angle between the two spins of $151.32^{\circ}$. The magnetic moments of the $\mathrm{C}$ atoms along the same edge are instead ferromagnetically coupled. We have thoroughly checked that the twisting of the moments stems from the substrate-induced SOC by rigidly shifting the GNR away from the $\mathrm{Sb}_{2} \mathrm{Te}_{3}$ slab and recomputing the magnetic properties (for starting magnetizations along $x, y$, or $z$ ). The tilting shows up only for sufficiently short GNR-substrate distances, at which there is significant chemical interaction between the two subsystems. Furthermore, noncollinear simulations without SOC yield an exact AFM configuration where the two moments form an angle of $180^{\circ}$. We have also carried out test simulations using a wider supercell along $y$, so as to double the distance between the periodic images of the GNR. Since it is not computationally feasible to study a 15-layer slab using this supercell, we have considered a slab consisting of a single quintuple layer. We 
(a)
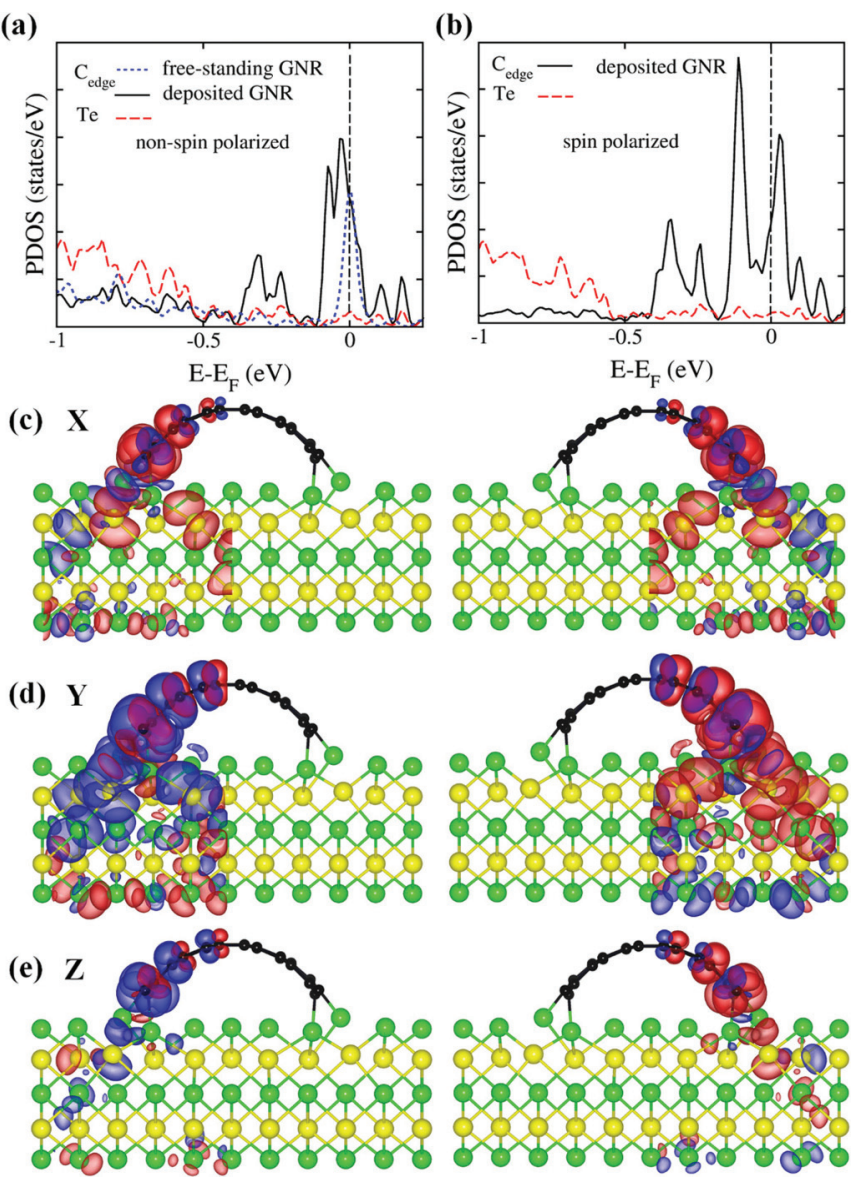

FIG. 2. Magnetic properties. (a) Nonspin-polarized and (b) spinpolarized PDOS for the $|j=3 / 2, l=1\rangle+|j=1 / 2, l=1\rangle$ spin-angle functions of the three edge $\mathrm{C}$ atoms of the GNR (black lines) and the nearest neighbor $\mathrm{Te}$ atoms (dashed red lines). The peak at $\mathrm{E}_{F}$ in (a) corresponds to the edge state. The PDOS in (b) correspond to the magnetic configuration shown in Fig. 1. The non-spin-polarized PDOS for the edge $\mathrm{C}$ atoms of a freestanding monohydrogenated GNR is also shown in (a) for the sake of comparison (dashed blue line). (c)-(e) Isovalue surfaces of the spin polarization density along $x$ (c), $y(\mathrm{~d})$, and $z$ (e) for the magnetic configuration in Fig. 1. The red (blue) surface indicates spin up (down) density, corresponding to $\pm 4 \times 10^{-5} \mu_{B} /$ (a.u. $)^{3}$. The plots clearly show that the $y$ and $z$ components form an AFM configuration across the GNR, whereas the $x$ components of the two edge spins are parallel.

have found that the twisting of the spins occurs in these five-layer models as well and that doubling the size of the cell does not affect the magnitude of this tilting significantly. The latter simulations show that the chiral interaction is indeed mediated by the GNR and is not an artifact due to the presence of the periodic images of the GNR.

\section{DISCUSSION}

To rationalize our findings, it is useful to map the system onto an effective spin Hamiltonian [44]. Such mapping has been carried out rigorously both for chiral GNRs [45], which possess edge states localized at the zigzag segments, and zigzag GNRs [46]. The resulting exchange coupling constants are FM along the edge and AFM across the edge. Furthermore, the intra-edge FM coupling is long-range for zigzag GNRs, which reflects the delocalized nature of the edge spin polarization in this geometry $[46,47]$. In these works, freestanding GNRs were considered and, thus, SOC was neglected. In our DFT models, the strong SOC induced by the substrate brings about a dependence of the energy on the spin direction, namely the magnetic anisotropy energy (MAE), as well as a DMI, due to the absence of inversion symmetry at the surface.

In principle, the DMI could be included in the effective Heisenberg Hamiltonian developed in Ref. [46] by taking into account the indirect exchange mechanism between the spins mediated by the surface-state conduction electrons, following an approach similar to Ref. [7]. Here, we provide a qualitative analysis of the main effects of the DMI. We consider a classical spin Hamiltonian, and assume that, along the edge, the strong, long-range FM coupling, combined with MAE effects, prevails over the DMI. This assumption is corroborated by our test simulations of chiral configurations along the edge. In these simulations, we have considered initial spin configurations corresponding to spin spirals with period length equal to three GNR units (commensurate with the supercell employed), in which the magnetic moments of nearest-neighbour spins form an angle of 120 degrees. All of these models converge to a FM configuration. It is computationally not feasible to study spin spiral configurations with longer period because this would require the use of supercells at least twice as large along the nanoribbon direction. Since the edge spin configuration is fully FM, one needs only to consider the DMI between pairs of nearest-neighbour spins on different edges. Hence, if only magnetic interactions up to second order are considered, the ground-state magnetic configuration is determined by the interplay between three terms:

$$
H=J S_{1} \cdot S_{2}+D \cdot S_{1} \times S_{2}+\sum_{i=1}^{2} S_{i}^{+} \cdot K \cdot S_{i},
$$

where $S_{1}$ and $S_{2}$ are the spins of two nearest-neighbour states on opposite edges. The first term is the isotropic exchange interaction between the two spins; here, $J>0$ is the exchange constant favoring the AFM coupling. The second term is the antisymmetric DMI, whose strength depends on the Dzyaloshinskii vector $\boldsymbol{D}$ (to be determined from the simulations). Finally, the third term describes the MAE for the two spins and $\boldsymbol{K}$ is the so-called anisotropy energy tensor.

Symmetry considerations [9] restrict the number of nonzero components of $\boldsymbol{D}$. The model possesses a mirror symmetry with respect to the $y z$ plane parallel to the GNR, which cuts the GNR into two halves [see Fig. 1(b)]. The freestanding GNR also possesses a mirror symmetry with respect to the perpendicular $x z$ plane. However, this symmetry is broken by the substrate, since the latter has $C_{3 v}$ symmetry and the edges of the GNR are parallel to one of its mirror planes. More precisely, in the geometry shown in Fig. 1, the first Te layer preserves the mirror symmetry, whereas the layers beneath break it. As a result, upon relaxation, the geometry of the GNR and the uppermost Te layer also deviates from a mirror-symmetric configuration. In summary, due to the $y z$ mirror plane, the vector $\boldsymbol{D}$ should lie in this plane (in the presence of an additional $x z$ mirror plane, the vector $\boldsymbol{D}$ would be parallel to $y$ ). As regards the anisotropy tensor for the edge 
TABLE I. Parameters $\boldsymbol{J}, \boldsymbol{D}$, and $\boldsymbol{K}$ of the spin Hamiltonian as obtained from the first-principles calculations. The values $\mathrm{K}_{1}, \mathrm{~K}_{2}$, and $K_{3}$ are the principal components of the tensor $\boldsymbol{K}$ in Eq. (2). They correspond to the magnetic anisotropies along the principal axes. The directions of the principal axes are also provided.

\begin{tabular}{c}
\hline \hline Exchange coupling $J$ and $\boldsymbol{D}$ vector \\
\hline$J=1.3 \mathrm{meV}$ \\
$D_{y}=0.7 \mathrm{meV}$ \\
$D_{z}=1.7 \mathrm{meV}$ \\
Principal components of $\boldsymbol{K}$ and principal axes \\
\hline $\mathrm{K}_{1}=0.0 \mathrm{meV},(0.633,0.085,-0.770)$ \\
$\mathrm{K}_{2}=1.2 \mathrm{meV},(0.056,0.996,-0.063)$ \\
$\mathrm{K}_{3}=3.6 \mathrm{meV},(0.772,-0.003,0.635)$ \\
\hline
\end{tabular}

spins, none of the components can be set to zero:

$$
\boldsymbol{D}=\left(\begin{array}{c}
0 \\
D_{y} \\
D_{z}
\end{array}\right), \boldsymbol{K}=\left(\begin{array}{l}
K_{x x} K_{x y} K_{x z} \\
K_{x y} K_{y y} K_{y z} \\
K_{x z} K_{y z} K_{z z}
\end{array}\right)
$$

Notice that, as far as the anisotropy of the total magnetization of the system is concerned, the corresponding tensor has zero $x y$ and $x z$ components, owing to the presence of the mirror plane $y z$.

The quantities $\boldsymbol{J}, \boldsymbol{D}$, and $\boldsymbol{K}$ can be estimated from a proper set of self-consistent simulations with different directions of the edge magnetizations. More specifically, the exchange coupling parameter $J$ is accurately obtained from the scalarrelativistic energy difference between the collinear FM and AFM configurations. Then, the three components of $\boldsymbol{D}$ are computed by considering pairs of configurations where the two spins lie on the plane perpendicular to the relevant component of $\mathrm{D}$ and form angles equal to +90 and -90 degrees, respectively. These calculations indicate that the main component of $\boldsymbol{D}$ is indeed on the $y z$ plane, although a non-negligible $x$ component is also found (see the Supplemental Material [41]). Finally, the six independent elements of the anisotropy tensor are obtained by fitting to the energy of the three selfcalculations discussed before and of additional constrained simulations. After evaluating the tensor, we diagonalize it to determine the principal values $\mathrm{K}_{1}, \mathrm{~K}_{2}$, and $\mathrm{K}_{3}$, which yield the magnetic anisotropies along the principal axes.

The values for $J, D, K_{1}, K_{2}$, and $K_{3}$ and the directions of the principal axes are shown in Table 1. The magnitude of the Dzyaloshinskii vector and the magnetic anisotropies are relatively large. Furthermore, the easy axis corresponding to $\mathrm{K}_{1}$ is practically in the $y z$ plane and forms an angle of about $40^{\circ}$ with respect to the $z$ axis. The MAE cannot account for the tilting effect observed in our models, which stems from the chiral DMI. In particular, tilting of the spins would also occur for an AFM configuration with starting edge magnetizations along the easy axis.

Finally, it is important to assess the robustness of the chiral spin configurations we find. Besides extrinsic effects [48,49], the one-dimensional nature of edge magnetism creates two types of intrinsic instabilities, which originate, respectively, from the quantum fluctuations due to the AFM interedge coupling [45,47] and from thermal fluctuations [50]. In our model, the strong substrate-induced magnetic anisotropy (of the order of meV) can partly stabilize the ferromagnetic polarization along the edge against thermal fluctuations, yielding a crossover temperature of the order of $100 \mathrm{~K}$ [50]. Furthermore, the long-range nature [46] of the intra-edge FM coupling in zigzag GNRs stabilizes edge magnetism against quantum fluctuations for sufficiently wide GNRs [47], contrary to the case of chiral nanoribbons [45].

\section{CONCLUSIONS}

In conclusion, we have found that a TI substrate induces noncollinear edge magnetism in GNRs via a robust DMI. This proximity effect stems from the strong SOC of the substrate. In principle, any substrate with large SOC could lead to such phenomenon, unless chemical interactions at the edge destabilize the edge magnetism. The latter effect is observed for some heavy metal substrates $[26,42]$. Notice that passivated GNRs on $\mathrm{Sb}_{2} \mathrm{Te}_{3}$ do not show any chiral magnetism owing to the large distance with respect to the substrate and the resulting weak effective SOC at the GNR [29]. From the application perspective, the finite magnetization originating from the twisting of the edge spins could be exploited in graphene-based spintronics devices [51-53]. In fact, there has recently been considerable experimental and theoretical work aimed at stabilizing the ferromagnetic state in GNRs, in view of potential applications as spin filters. In many respects, our system is simpler to realize than alternative approaches, which either require edge modification (a complex chemical process) $[54,55]$ or the application of a very strong electric field [24].

\section{ACKNOWLEDGMENTS}

We acknowledge useful discussions with S. Blügel, G. Bihlmayer, M. Schmidt, S. Wessel, and Y. Li. We also acknowledge the computational resources provided by JARAHPC from RWTH Aachen University under Project No. JARA0107 and the support by HPCC platform of Xi'an Jiaotong University, as well as the support of the National Supercomputing Center in Shenzhen. W.Z. gratefully thanks the support of the Youth Thousand Talents Program of China and the Young Talent Support Plan of Xi'an Jiaotong University. F.H. acknowledges funding by the DFG (German Science Foundation) under Grant No. RTG 1995.

W.Z. and F.H. contributed equally to this work.
[1] L. Fu, C. L. Kane, and E. J. Mele, Phys. Rev. Lett. 98, 106803 (2007)

[2] J. E. Moore and L. Balents, Phys. Rev. B 75, 121306(R) (2007).

[3] R. Roy, Phys. Rev. B 79, 195322 (2009).
[4] M. Z. Hasan and C. L. Kane, Rev. Mod. Phys. 82, 3045 (2010).

[5] X. L. Qi and S. C. Zhang, Rev. Mod. Phys. 83, 1057 (2011).

[6] Q. Liu, C. X. Liu, C. K. Xu, X. L. Qi, and S. C. Zhang, Phys. Rev. Lett. 102, 156603 (2009). 
[7] F. Ye, G. H. Ding, H. Zhai, and Z. B. Su, Europhys. Lett. 90, 47001 (2010).

[8] I. E. Dzyaloshinskii, Sov. Phys. JETP 5, 1259 (1957).

[9] T. Moriya, Phys. Rev. 120, 91 (1960).

[10] M. Bode, M. Heide, K. von Bergmann, P. Ferriani, S. Heinze, G. Bihlmayer, A. Kubetzka, O. Pietzsch, S. Blügel, and R. Wiesendanger, Nature 447, 190 (2007).

[11] M. Menzel, Y. Mokrousov, R. Wieser, J. E. Bickel, E. Vedmedenko, S. Blügel, S. Heinze, K. von Bergmann, A. Kubetzka, and R. Wiesendanger, Phys. Rev. Lett. 108, 197204 (2012).

[12] R. Mazzarello and E. Tosatti, Phys. Rev. B 79, 134402 (2009).

[13] A. A. Khajetoorians, M. Steinbrecher, M. Ternes, M. Bouhassoune, M. D. Dias, S. Lounis, J. Wiebe, and R. Wiesendanger, Nat. Commun. 7, 10620 (2016).

[14] J. G. Checkelsky, J. T. Ye, Y. Onose, Y. Iwasa, and Y. Tokura, Nat. Phys. 8, 729 (2012).

[15] Y. L. Chen, J.-H. Chu, J. G. Analytis, Z. K. Liu, K. Igarashi, H.-H. Kuo, X. L. Qi, S. K. Mo, R. G. Moore, D. H. Lu, M. Hashimoto, T. Sasagawa, S. C. Zhang, I. R. Fisher, Z. Hussain, and Z. X. Shen, Science 329, 659 (2010).

[16] F. Katmis, V. Lauter, F. S. Nogueira, B. A. Assaf, M. E. Jamer, P. Wei, B. Satpati, J. W. Freeland, I. Eremin, D. Heiman, P. Jarillo-Herrero, and J. S. Moodera, Nature 533, 513 (2016).

[17] O. A. Pankratov, Phys. Lett. A 121, 360 (1987).

[18] M. Fujita, K. Wakabayashi, K. Nakada, and K. Kusakabe, J. Phys. Soc. Jpn. 65, 1920 (1996).

[19] Y. W. Son, M. L. Cohen, and S. G. Louie, Phys. Rev. Lett. 97, 216803 (2006).

[20] L. Y. Jiao, L. Zhang, X. R. Wang, G. Diankov, and H. J. Dai, Nature 458, 877 (2009).

[21] J. M. Cai, P. Ruffieux, R. Jaafar, M. Bieri, T. Braun, S. Blankenburg, M. Muoth, A. P. Seitsonen, M. Saleh, X. Feng, K. Müllen, and R. Fasel, Nature 466, 470 (2010).

[22] X. L. Li, X. R. Wang, L. Zhang, S. W. Lee, and H. J. Dai, Science 319, 1229 (2008).

[23] P. Ruffieux, S. Wang, B. Yang, C. Sánchez-Sánchez, J. Liu, T. Dienel, L. Talirz, P. Shinde, C. A. Pignedoli, D. Passerone, T. Dumslaff, X. Feng, K. Müllen, and R. Fasel, Nature 531, 489 (2016).

[24] Y. W. Son, M. L. Cohen, and S. G. Louie, Nature 444, 347 (2006).

[25] D. Hsieh, Y. Xia, D. Qian, L. Wray, F. Meier, J. H. Dil, J. Osterwalder, L. Patthey, A. V. Fedorov, H. Lin, A. Bansil, D. Grauer, Y. S. Hor, R. J. Cava, and M. Z. Hasan, Phys. Rev. Lett. 103, 146401 (2009).

[26] Y. Li, W. Zhang, M. Morgenstern, and R. Mazzarello, Phys. Rev. Lett. 110, 216804 (2013).

[27] G. Z. Magda, X. Z. Jin, I. Hagymasi, P. Vancso, Z. Osvath, P. Nemes-Incze, C. Y. Hwang, L. P. Biro, and L. Tapaszto, Nature 514, 608 (2014).

[28] C. G. Tao, L. Jiao, O. V. Yazyev, Y.-C. Chen, J. Feng, X. Zhang, R. B. Capaz, J. M. Tour, A. Zettl, S. G. Louie, H. Dai, and M. F. Crommie, Nat. Phys. 7, 616 (2011).

[29] W. Zhang, F. Hajiheidari, Y. Li, and R. Mazzarello, Sci. Rep. 6, 29009 (2016).
[30] K. H. Jin and S. H. Jhi, Phys. Rev. B 87, 075442 (2013).

[31] J. Zhang, C. Triola, and E. Rossi, Phys. Rev. Lett. 112, 096802 (2014).

[32] C. D. Spataru and F. Léonard, Phys. Rev. B 90, 085115 (2014).

[33] M. Gmitra and J. Fabian, Phys. Rev. B 92, 155403 (2015).

[34] M. Gmitra, D. Kochan, P. Högl, and J. Fabian, Phys. Rev. B 93, 155104 (2016).

[35] P. Giannozzi, S. Baroni, N. Bonini, M. Calandra, R. Car, C. Cavazzoni, D. Ceresoli, G. L. Chiarotti, M. Cococcioni, I. Dabo, A. D. Corso, S. Fabris, G. Fratesi, S. de Gironcoli, R. Gebauer, U. Gerstmann, C. Gougoussis, A. Kokalj, M. Lazzeri, L. Martin-Samos, N. Marzari, F. Mauri, R. Mazzarello, S. Paolini, A. Pasquarello, L. Paulatto, C. Sbraccia, S. Scandolo, G. Sclauzero, A. P. Seitsonen, A. Smogunov, P. Umari, and R. M. Wentzcovitch, J. Phys.: Condens. Matter 21, 395502 (2009).

[36] J. P. Perdew, K. Burke, and M. Ernzerhof, Phys. Rev. Lett. 77, 3865 (1996).

[37] S. Grimme, J. Comput. Chem. 27, 1787 (2006).

[38] J. P. Perdew and A. Zunger, Phys. Rev. B 23, 5048 (1981).

[39] S. Heinze, K. von Bergmann, M. Menzel, J. Brede, A. Kubetzka, R. Wiesendanger, G. Bihlmayer, and S. Blügel, Nat. Phys. 7, 713 (2011).

[40] H. J. Monkhorst and J. D. Pack, Phys. Rev. B 13, 5188 (1976).

[41] See Supplemental Material at http://link.aps.org/supplemental/ 10.1103/PhysRevB.96.245413 for additional information about bonding mechanisms and magnetic properties of the models and for the description of the structural properties of the stable and metastable models of GNRs on $\mathrm{Sb}_{2} \mathrm{Te}_{3}$.

[42] Y. Li, D. Subramaniam, N. Atodiresei, P. Lazić, V. Caciuc, C. Pauly, A. Georgi, C. Busse, M. Liebmann, S. Blügel, M. Pratzer, M. Morgenstern, and R. Mazzarello, Adv. Mater. 25, 1967 (2013).

[43] Y. Li and R. Mazzarello, Phys. Rev. B 88, 045317 (2013).

[44] T. Hikihara, X. Hu, H.-H. Lin, and C.-Y. Mou, Phys. Rev. B 68, 035432 (2003).

[45] M. Golor, S. Wessel, and M. J. Schmidt, Phys. Rev. Lett. 112, 046601 (2014).

[46] C. Koop and M. J. Schmidt, Phys. Rev. B 92, 125416 (2015).

[47] C. Koop and S. Wessel, Phys. Rev. B 96, 165114 (2017).

[48] T. Wassmann, A. P. Seitsonen, A. M. Saitta, M. Lazzeri, and F. Mauri, Phys. Rev. Lett. 101, 096402 (2008).

[49] J. Kunstmann, C. Özdogan, A. Quandt, and H. Fehske, Phys. Rev. B 83, 045414 (2011).

[50] O. V. Yazyev and M. I. Katsnelson, Phys. Rev. Lett. 100, 047209 (2008).

[51] W. Han, R. K. Kawakami, M. Gmitra, and J. Fabian, Nat. Nanotechnol. 9, 794 (2014).

[52] D. Pesin and A. H. MacDonald, Nat. Mater. 11, 409 (2012).

[53] S. Roche et al., 2D Mater. 2, 030202 (2015).

[54] E. J. Kan, Z. Y. Li, J. L. Yang, and J. G. Hou, J. Am. Chem. Soc. 130, 4224 (2008).

[55] S. Dutta, A. K. Manna, and S. K. Pati, Phys. Rev. Lett. 102, 096601 (2009). 